\title{
Représentations des enseignants sur leur métier et acquisition de L2 de la part des apprenants : quels liens peuvent être établis?
}

\section{Rebecca Starkey-Perret}

\section{(2) OpenEdition}

Édition électronique

URL : http://journals.openedition.org/rdlc/1563

DOI : $10.4000 /$ rdlc. 1563

ISSN : 1958-5772

Éditeur

ACEDLE

\section{Référence électronique}

Rebecca Starkey-Perret, « Représentations des enseignants sur leur métier et acquisition de L2 de la part des apprenants : quels liens peuvent être établis ? ", Recherches en didactique des langues et des cultures [En ligne], 10-1 | 2013, mis en ligne le 07 janvier 2013, consulté le 20 avril 2019. URL : http:// journals.openedition.org/rdlc/1563; DOI : 10.4000/rdlc.1563

\section{Ce document a été généré automatiquement le 20 avril 2019}

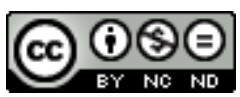

Recherches en didactique des langues et des cultures is licensed under a Creative Commons AttributionNonCommercial-NoDerivatives 4.0 International License 


\title{
Représentations des enseignants sur leur métier et acquisition de L2 de la part des apprenants : quels liens peuvent être établis?
}

\author{
Rebecca Starkey-Perret
}

\section{Introduction}

1 Les travaux de Narcy-Combes, J.-P., Narcy-Combes, M.-F. et Starkey-Perret (2009) et de Starkey-Perret (2011), entre autres, ont permis de mettre en lumière les représentations que des professeurs de langue dans le secondaire en France construisent sur leur métier et sur les apprenants auprès de qui ils l'exercent. Les résultats les plus significatifs montrent que la majorité des répondants estimaient s'investir de manière conséquente dans leur métier pour un retour symbolique et matériel insuffisant à leurs yeux. Ils ne se sentaient pas préparés par les concours de recrutement et par la formation pour la réalité $\mathrm{du}$ terrain, notamment pour travailler avec les apprenants qu'ils qualifiaient de consommateurs $(37,3 \%)$, de déconcentrés $(30,9 \%)$ et de passifs $(23,6 \%)$, et qui, selon certains, manquent de culture / connaissances (23,6\%), sont démotivés (18,2\%), ou bien paresseux (16,8\%) (Starkey-Perret, 2011). Finalement, les enquêtes indiquent qu'une vision transmissive de l'apprentissage / enseignement des langues perdure pour $88 \%$ des répondants (Starkey-Perret, 2011).

2 La présente contribution propose d'aller plus loin en examinant la manière dont ces représentations influent sur l'action de terrain, en étudiant les choix pédagogiques et les attitudes d'un nombre restreint d'enseignantes ( 5 volontaires). Grâce à une triangulation des observations, des discours des acteurs eux-mêmes après l'observation et des théories de l'acquisition et de la psychologie de l'éducation, des liens entre les représentations, la pédagogie, les attitudes induites et les potentialités d'acquisition pourront être établis. 


\section{Appuis théoriques}

\section{Théories de l'acquisition}

3 La vision de l'acquisition retenue ici propose de concilier les théories socioconstructivistes et cognitivistes. Elle prend donc en compte les apports des neuroscientifiques, des interactionnistes et de ceux qui étudient de plus près le rôle que jouent les affects dans l'investissement que les individus consentent à mettre dans leur apprentissage. Dans une perspective complexe (Morin, 2000), ces théories sont interdépendantes.

4 Le paradigme cognitiviste et connexionniste de l'acquisition montre que plus un item d'input est fréquent, plus les schémas de connexions entre différents neurones sont renforcés. Ainsi, un comportement de plus en plus conforme aux normes de la langue cible peut être produit en fonction de la fréquence de l'input (Ellis, 2003). Ce constat connexionniste ne conduit pas à nier l'importance de la production des apprenants pour l'acquisition, telle qu'elle est montrée par les travaux de Swain (2000), mais renforce la préconisation de pratiques lors desquelles l'enseignant serait amené à fournir un maximum d'input lors des cours.

5 Le positionnement constructiviste soutient que le sens et le savoir sont construits par les apprenants lors de l'interaction. La notion de Zone Proximale de Développement (ZPD) de Vygotsky (1978) confirme l'importance de l'interaction pour l'apprentissage. Le potentiel d'apprentissage est majoré lorsque l'apprenant travaille en collaboration avec des pairs plus expérimentés ou avec un expert (ici, l'enseignant). Pour Swain (2000), la coconstruction que permet l'interaction consiste en la résolution de problèmes à travers le dialogue. Ce dialogue dit collaboratif (Swain, 2000), permet d'attirer l'attention de l'apprenant sur ses difficultés langagières, le conduisant à construire des hypothèses et à les tester, ce qui induit une métaréflexion linguistique (Little, 2007). Il s'agit d'une négociation de sens, définie par Pica (1994) comme la modification et la restructuration de l'interaction lorsque les apprenants et leurs interlocuteurs ont des difficultés d'intercompréhension. Cette négociation de sens (le fond) dans l'interaction permet également de se focaliser sur la forme (Lightbown \& Spada, 1990 ; Doughty \& Williams, 1998).

6 Swain (1995) souligne l'importance de la production, qui amène au traitement en profondeur du langage, car elle demande davantage d'efforts et de contrôle que le traitement de l'input. C'est donc la production dans un objectif communicationnel précis qui permet à l'apprenant de prendre conscience de ce qu'il est capable d'exprimer en L2 (Swain, 2000). Elle favorise également le repérage, c'est-à-dire la capacité de l'apprenant à remarquer l'écart entre ce qu'il souhaiterait dire et ce qu'il est capable de dire, ce qui lui permet d'ajuster ses hypothèses (Schmidt \& Frota, 1986). Suite au repérage, les apprenants pourront mieux cibler des éléments dans l'input qui permettent de remédier aux écarts (gaps, Jourdenais, 2001). De plus, lorsque les apprenants sont amenés à prendre la parole par le biais de l'interaction, ils ont davantage d'opportunités de pratiquer, ce qui permet l'ancrage en mémoire des connaissances et leur automatisation (Johnstone, Ashbaugh \& Warfield, 2002).

7 Dans le paradigme interactionniste de l'acquisition, l'enseignant joue un rôle crucial par le biais des rétroactions correctives. Si une composante-clé de l'apprentissage est le 
repérage (Schmidt, 1990), il s'ensuit qu'une manière d'éviter la fossilisation des productions non conformes aux attentes (désormais PNCA, Narcy-Combes, J.-P. \& NarcyCombes, M-F., 2007) est d'attirer l'attention de l'apprenant sur ces formes et de l'encourager à émettre d'autres hypothèses. Les travaux de Chaudron (1988), de Lyster et Ranta (1997) et de Mackey, Oliver et Leeman (2003) ont montré que "la capacité des apprenants à modifier leur output dans la réponse à la rétroaction tend à indiquer qu'ils ont utilisé en quelque façon cette rétroaction" (Cosereanu-Declerck, 2010: 90). Ainsi, l'autocorrection ( student generated repair) en réponse à la rétroaction indiquerait un haut de degré d'incorporation (uptake) de la part de l'apprenant, car il est amené à focaliser son attention sur la forme qui lui pose problème et à apporter lui-même une réponse, au lieu de tout simplement répéter une forme déjà fournie. En s'appuyant sur les travaux de Chaudron (1988), de Doughty (1994) et de Spada et Fröhlich (1995), Lyster et Ranta (1997) ont établi une typologie des rétroactions correctives de l'enseignant. Ensuite, ils ont fait un lien entre le type de rétroaction fournie par des enseignants et le degré d'incorporation induite. Les 6 types de rétroaction identifiés dans l'étude de Lyster et Ranta (1997, pp. 46 -49) sont les suivants :

- La correction explicite (explicit correction): l'enseignant fournit la forme attendue en indiquant clairement que la production de l'apprenant n'était pas conforme ;

- La reformulation implicite (recast): l'enseignant reformule une partie ou la totalité de l'énoncé de l'apprenant en omettant la production non conforme. L'enseignant ne signale pas que la production de l'apprenant n'était pas conforme. Souvent l'enseignant incorpore la forme attendue tout en continuant la communication. Les traductions de L1 à L2 sont incluses dans cette catégorie ;

- La demande de clarification (clarification request) : l'enseignant indique que l'énoncé n'a pas été compris et qu'une répétition ou une reformulation est nécessaire ;

- L'indice métalinguistique (metalinguistic clues): l'enseignant fait un commentaire concernant la forme de l'énoncé qui indique que celui-ci n'est pas conforme. (ex. "problem", "pronunciation", "there's an error", "no", "is it plural ?);

- L'incitation (elicitation) : l'enseignant utilise trois techniques pour inciter l'apprenant à utiliser la forme attendue. Premièrement, l'enseignant recommence l'énoncé et fait une pause pour permettre à l'apprenant de le compléter. Deuxièmement, l'enseignant pose une question telle que "comment dit-on ?" Troisièmement, l'enseignant demande à l'apprenant de reformuler son énoncé ;

- La répétition (repetition) : l'enseignant répète la PNCA en l'isolant et en ajustant son intonation afin d'attirer l'attention sur l'énoncé.

Les résultats de Lyster et Ranta (1997), qui ont un échantillon similaire à celui étudié ici en termes de nombre d'enseignants et de nombre d'heures observés, mais dans un contexte différent, ont montré que la reformulation implicite, bien que ce soit la forme de rétroaction préférée des enseignants observés, est le type de rétroaction qui amène le moins à l'incorporation chez l'apprenant. La reformulation implicite ainsi que la correction implicite ne peuvent pas induire l'autocorrection ni l'hétéro-correction, puisqu'il s'agit de types de rétroaction lors desquelles l'enseignant fournit la forme attendue. 


\section{Théories de la psychologie de l'éducation}

9 Certains travaux en psychologie de l'éducation visent à intégrer le rôle des affects dans l'apprentissage. Les théories de l'attribution (Weiner, 1992) et de l'expectancy-value (Eccles et al., 1983) ainsi que la théorie du contrôle et de la valeur des émotions d'accomplissement (Pekrun et al., 2007) mettent en avant, entre autres, l'importance de la croyance de l'apprenant dans ses capacités à accomplir des tâches d'apprentissage. S'il attribue un échec, ou les PNCA, à son intelligence, il est probable qu'il investisse de moins en moins d'énergie et de temps dans les activités proposées. La valeur que l'apprenant accorde à la matière enseignée et aux activités d'apprentissage ainsi que l'environnement affectif mis en place par l'enseignant ont également un impact sur l'investissement des apprenants (Williams \& Burden, 1997).

10 Ainsi, l'enseignant joue un rôle prépondérant dans l'investissement des apprenants (Goddard, Hoy \& Hoy, 2004) par le biais de la valorisation ou de la dévalorisation des apprenants. Selon la psychologie de l'éducation, l'enseignant valorise les apprenants en mettant en place un environnement de confiance et de tolérance (Brophy, 198 ; Bandura, 1986) dans laquelle l'enseignant salue les apprenants lorsqu'ils entrent dans la pièce, leur sourit, apprend et utilise leurs prénoms, et montre un intérêt pour ce qui les motive, par exemple (Burden, 1995; Raffini, 1996). Il les valorise en les encourageant et en leur donnant du renforcement positif (Bandura, 1997), tout en proposant des rétroactions réalistes sur leurs productions (Försterling \& Morgenstern, 2002) accompagnées de pistes de travail individuelles (Demaizière \& Narcy-Combes, J.-P., 2005; Robinson, 2002). L'importance de la sincérité des renforcements positifs est soulignée. Les recherches montrent que les apprenants font aisément la différence entre des automatismes et les encouragements sincères (Schunk, Pintrich \& Meece, 2008).

11 L'importance de la communication des attentes élevées (mais réalistes) pour les apprenants (Pressley \& McCormick, 1995) a été montrée par l'effet Pygmalion (Rosenthral \& Jacobson, 1968; Felouzis, 1997), c'est-à-dire l'ajustement des performances des apprenants pour répondre aux attentes des enseignants. L'enseignant dévalorise les apprenants en oubliant leurs prénoms ou en ne prenant pas le temps de les apprendre, ni de s'intéresser à eux, en encourageant un haut degré de compétitivité entre les apprenants (Perry et al., 1994), en se moquant de leurs productions (Wigfield, Eccles \& Pintrich, 1996) et en exposant leur vie privée devant les autres (Dubet \& Martuccelli, 1996). Les apprenants qui se sentent dévalorisés courent un risque accru de se désinvestir.

\section{Les représentations des enseignants}

12 La position soutenue ici est qu'il ne suffit pas de dire à un enseignant qu'il faudrait qu'il agisse de telle ou telle manière pour que cela se fasse. En effet, les choix pédagogiques et les attitudes des enseignants sont influencés par les représentations qu'ils construisent sur eux-mêmes, sur l'apprentissage, sur le langage et sur les apprenants (Starkey-Perret et al., 2012).

13 Les représentations que l'enseignant construit sur l'apprentissage et sur son rôle et les attitudes et postures induites font émerger la nature complexe de son investissement. $\mathrm{Si}$ les représentations que l'enseignant construit sur l'acquisition ne sont pas en cohérence 
avec les théories de l'acquisition, il est probable qu'il ne mettra pas en place une pédagogie favorable à l'acquisition. Néanmoins, il peut avoir des intuitions qui vont dans le sens des théories (Elias, 1993). Un enseignant qui a une vision transmissive de l'acquisition a plus de chances de mettre en place un environnement centré sur l'enseignant. Un enseignant pour qui l'apprentissage d'une langue se résume à la mémorisation de règles grammaticales mettra probablement en place une pédagogique qui prône l'apprentissage des règles, peut-être au détriment de la communication. Ainsi, la prise en compte des représentations des enseignants est primordiale pour comprendre leur action sur le terrain.

14 La considération des principes d'investissement et de retour sur investissement tels qu'ils sont théorisés par Norton Pierce (1995) souligne l'importance des retours sur investissement, qu'ils soient d'ordre symbolique ou matériel, pour stimuler l'investissement. Cette théorisation est particulièrement applicable aux enseignants. Il est postulé ici que les retours sur investissement joueront un rôle prépondérant car ces retours sont également alliés à la valeur que l'enseignant voit accorder à son métier par la société, ce qui n'est pas anodin pour le développement de son identité professionnelle. On constate qu'en effet, tous ces facteurs se lient dans un ensemble complexe et dynamique.

\section{Le contexte}

15 La perspective complexe des systèmes d'apprentissage conduit également à considérer l'impact des contextes dans l'enseignement. Le macrocontexte éducatif français tel qu'il est décrit par les sociologues de l'éducation montre que le statut des enseignants au sein de la société a changé depuis l'école de la 3ème République. Puisque l'école d'aujourd'hui joue un rôle pivot dans la stratification sociale des individus, elle est plus ouverte aux critiques des divers membres de la société (Dubet, 2008). La massification scolaire a modifié la position de l'école dans la société en lui donnant la tâche de sélectionner les individus et de les orienter vers leur futur emploi (Duru-Bellat, 2006). Le résultat est la hiérarchisation des établissements et des filières, ce qui induit, pour un grand nombre d'individus, un sentiment d'échec. Dès lors, les apprenants concernés, c'est-à-dire une grande partie du public, se sentent méprisés et peuvent, dans le pire des cas, se sentir en situation de révolte contre l'école (Dubet, 2008) ou se résigner à l'échec scolaire. (Sternberg \& Williams, 2010 ; voir aussi supra paragraphe 2.2).

La massification scolaire a eu pour conséquence qu'une place grandissante a été faite aux cultures juvéniles au sein de l'école (Dubet \& Martuccelli, 1996). Celles-ci sont, en majeure partie, éloignées de la culture scolaire valorisée et véhiculée par le corps professoral (Felouzis, 1997). Le changement des publics induit un changement de rôle pour les enseignants. Leurs connaissances disciplinaires ne suffisent plus pour conquérir les élèves. On attend d'eux qu'ils mettent en place les conditions de l'apprentissage au travers d'une relation pédagogique qui reconnait et valorise les élèves en tant qu'individus (Dubet, 2008). Si un enseignant ne se reconnait pas dans ces nouveaux rôles, des tensions peuvent en résulter, ce qui pourra au bout du compte entraver l'acquisition des compétences visées. 


\section{Questions de recherche}

17 Les références théoriques exposées ici donnent des pistes de réflexion pour les praticiens. Elles ont également montré que la mise en place des préconisations issues des théories n'est pas si simple que cela. De ce constat découlent les questions suivantes :

QR1 : Les enseignantes qui participent à cette étude mettent-elles en place des pratiques cohérentes avec les théories retenues?

QR2 : Quels sont les facteurs représentationnels qui ont le plus d'impact sur leurs choix pédagogiques et sur les attitudes qu'elles ont envers les apprenants?

QR3 : Si les représentations construites induisent des pédagogies qui vont à l'encontre des théories, de quelle manière peut-on envisager de les déstabiliser et de permettre leur évolution?

\section{Méthodologie}

21 Suite à une enquête par questionnaire (Starkey-Perret, 2011), des répondants ont été sélectionnés pour participer à l'observation de terrain. L'échantillonnage par le biais de la méthode des quotas a été préféré. Dans la méthode des quotas, répandue dans les recherches qualitatives, les individus ne sont pas tirés au sort, mais choisis selon leur conformité aux critères sélectionnés (De Ribaupierre, Ghisletta, \& Lecerf, 2010). Ainsi les 83 répondants qui avaient répondu positivement pour un entretien avec l'enquêteur ont été triés en fonction de l'intensité de leurs réponses portant sur leur satisfaction pour leur métier et en fonction de leurs réponses aux variables indépendantes afin d'assurer la représentativité en ce qui concerne le nombre de femmes et d'hommes, d'agrégés et de certifiés et de ceux qui exercent en collège et en lycée. Ce tri a permis de sélectionner 20 répondants qui ont été contactés par mail. Cette démarche a permis de trouver 2 volontaires (Sophie ${ }^{1} \&$ Nathalie, tableau 1 ).

22 Afin d'augmenter le nombre de participants, le champ de recherche a été élargi pour inclure tous les répondants qui ont exprimé leur accord pour un entretien, basculant ainsi de la méthode d'échantillonnage des quotas à une méthode entièrement volontariste. Cette démarche a permis de trouver 3 personnes de plus. Ainsi, quelques déséquilibres sont à noter dans l'échantillon, dans le tableau 1. Toutes sont des femmes certifiées dont 4 âgées de moins de 30 ans et une autre de 48 ans au moment de l'enquête. 3 personnes exercent dans des collèges et 2 dans des lycées, respectant ainsi l'équilibre des affectations dans ce sens. Néanmoins, les participantes exercent toutes dans des établissements de type ZEP ou dans des lycées offrant des parcours professionnels. Il est donc postulé que la place accordée aux discours sur la formation initiale et sur les publics sera accrue et que les éléments contextuels décrits dans le paragraphe 2.4 auront davantage de poids.

23 Au total 22 séances d'anglais ont été observées. Les observations ont été retranscrites et analysées en fonction du cadre théorique retenu (cf. supra paragraphes 2.1 et 2.2). Les analyses des observations qui font l'objet de cette contribution concernent le temps de parole en langue cible (en l'occurrence, l'anglais), le temps de parole des apprenants, les rétroactions correctives fournies par les volontaires, l'utilisation des prénoms des 
apprenants et les attitudes envers les apprenants qualifiées de "valorisantes" et de "dévalorisantes".

Suite aux observations, des entretiens individuels ont été menés avec les participantes. En raison des contraintes de temps des enseignantes observées, les entretiens postobservatoires ont eu lieu immédiatement après l'observation. Ainsi l'entretien a été préparé en amont des observations, à l'aide d'une grille d'observations. L'objectif des entretiens était de recueillir les impressions que les volontaires avaient des séances et des explications qu'elles donnaient pour leurs actions, afin de faire ressortir le lien entre les représentations et leurs choix. Ces entretiens ont été retranscrits intégralement et ont fait l'objet d'une analyse de contenu (Bardin, 2007).

Suite à ce travail, les résultats individuels des analyses ont été envoyés par courriel aux participantes avec des explications théoriques et terminologiques afin de leur permettre de réagir. Dans les résultats, les commentaires des participantes lors de l'entretien sont séparés des réactions données par courriel.

\section{Résultats}

Dans cette partie les résultats seuls sont exposés. Ils seront commentés et interprétés lors de la discussion.

\section{Le corpus}

Tableau 1 - Description du corpus de l'observation.

\begin{tabular}{|c|c|c|c|l|l|}
\hline Enseignante & Académie & $\begin{array}{c}\text { Type } \\
\text { d'Établissement }\end{array}$ & $\begin{array}{c}\text { Nombre } \\
\text { de } \\
\text { séances } \\
\text { observées }\end{array}$ & $\begin{array}{c}\text { Les } \\
\text { groupes } \\
\text { observés }\end{array}$ & $\begin{array}{c}\text { Effectifs } \\
\text { par } \\
\text { groupe }\end{array}$ \\
\hline Nathalie & Créteil & Collège ZEP & 5 & $\begin{array}{l}-2 \\
\text { groupes } \\
\text { 3ème } \\
-2 \\
\text { groupes } \\
4 \text { ème } \\
-1 \text { groupe } \\
\text { CLA }\end{array}$ & $\begin{array}{l}19.23 \\
17\end{array}$ \\
\hline
\end{tabular}




\begin{tabular}{|c|c|c|c|c|c|}
\hline Corinne & Créteil & $\begin{array}{l}\text { Lycée Technique } \\
\text { et professionnel }\end{array}$ & 6 & $\begin{array}{l}-1 \text { groupe } \\
2 \text { nde } \\
-2 \\
\text { groupes } \\
\text { 1ère } \\
-1 \text { groupe } \\
\text { terminale } \\
-2 \\
\text { groupes } \\
\text { BTS }\end{array}$ & \begin{tabular}{|l}
20 \\
30,6 \\
12 \\
19,20
\end{tabular} \\
\hline Anne & Créteil & Collège ZEP & 3 & $\begin{array}{l}-1 \text { groupe } \\
\text { 5ème } \\
-1 \text { groupe } \\
\text { 6ème } \\
\text { SEGPA } \\
\text {-1 groupe } \\
\text { 6ème }\end{array}$ & $\begin{array}{l}20 \\
11 \\
22\end{array}$ \\
\hline Danielle & Créteil & Collège ZEP & 4 & \begin{tabular}{|l}
-2 \\
groupes \\
de 3ème \\
-2 \\
groupes \\
de 6ème
\end{tabular} & $\begin{array}{l}17,17 \\
20,20\end{array}$ \\
\hline Julie & Reims & $\begin{array}{l}\text { Lycée technique } \\
\text { et professionnel }\end{array}$ & 4 & $\begin{array}{l}-1 \text { groupe } \\
2 \text { nde } \\
-3 \text { groupes } \\
1 \text { ère }\end{array}$ & $\begin{array}{l}13, \\
21,13 \\
11\end{array}$ \\
\hline Totaux & $\begin{array}{l}4 \text { Créteil } \\
1 \text { Reims }\end{array}$ & $\begin{array}{c}3 \text { collèges } \\
2 \text { lycées } \\
\text { techniques et } \\
\text { professionnels }\end{array}$ & 22 & $\begin{array}{l}-4 \text { 6ème } \\
-1 \text { 5ème } \\
-2 \text { 4ème } \\
-4 \text { 3ème } \\
- \text { Une } \\
\text { CLA } \\
-2 \text { 2nde } \\
-51 \text { ère } \\
-1 \\
\text { terminale } \\
-2 \text { BTS }\end{array}$ & $\begin{array}{lll}\text { De } & 6 & \text { à } \\
30 & & \end{array}$ \\
\hline
\end{tabular}

\section{Le temps de parole en anglais}

Tableau 2 - Répartition du temps de parole en anglais et en français dans les observations.

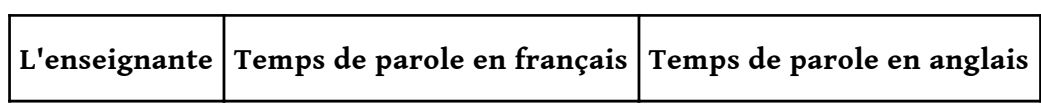




\begin{tabular}{|c|c|c|}
\hline Nathalie & $78 \%$ & $22 \%$ \\
\hline Corinne & $49 \%$ & $51 \%$ \\
\hline Anne & $33 \%$ & $67 \%$ \\
\hline Danielle & $54 \%$ & $46 \%$ \\
\hline Julie & $31,2 \%$ & $68,8 \%$ \\
\hline
\end{tabular}

\section{Le temps de parole des apprenants et de l'enseignante}

Tableau 3 - Répartition du temps de parole de l'enseignante et des apprenants dans les observations.

\begin{tabular}{|c|c|c|}
\hline L'enseignant & Temps de parole professeur & Temps de parole apprenants \\
\hline Nathalie & $38,9 \%$ & $61,1 \%$ \\
\hline Corinne & $60,6 \%$ & $39,4 \%$ \\
\hline Anne & $48,5 \%$ & $51,5 \%$ \\
\hline Danielle & $53,2 \%$ & $46,8 \%$ \\
\hline Julie & $65 \%$ & $35 \%$ \\
\hline
\end{tabular}

\section{Les rétroactions correctives}

Dans l'ensemble, $26 \%$ des 635 rétroactions ont déclenché des réparations générées par les apprenants (tableau 4). Les répétitions donnent lieu au taux le plus élevé de réparations générées par les apprenants dans cet échantillon contrairement à l'incitation dans l'échantillon de Lyster \& Ranta (1997). Les répétitions représentent $6 \%$ des rétroactions.

Tableau 4 - Récapitulatif des analyses des rétroactions correctives et réparations des PNCA de l'ensemble des participantes à l'enquête par observation.

\begin{tabular}{|l|l|l|l|l|}
\hline $\begin{array}{l}\text { Type de } \\
\text { rétroaction }\end{array}$ & Incorporation & Autoréparation & $\begin{array}{l}\text { Réparation } \\
\text { générée } \\
\text { par } \\
\text { d'autres } \\
\text { élèves }\end{array}$ & $\begin{array}{l}\text { Totales } \\
\text { réparations } \\
\text { générées } \\
\text { par les } \\
\text { apprenants }\end{array}$ \\
\hline
\end{tabular}




\begin{tabular}{|c|c|c|c|c|}
\hline $\begin{array}{l}\text { Reformulation } \\
\text { implicite } \\
(n=275) \\
43,3 \% \text { des } \\
\text { rétroactions }\end{array}$ & $59(21,5 \%)$ & 0 & 0 & $0 \%(n=0)$ \\
\hline $\begin{array}{ll}\text { Incitation } & (\mathrm{n}= \\
185) & \\
29 \% & \text { des } \\
\text { rétroactions } & \end{array}$ & 0 & $30(16,2 \%)$ & $66(35,7 \%)$ & $\begin{array}{l}52 \% \\
(n=96)\end{array}$ \\
\hline $\begin{array}{l}\text { Indice } \\
\text { métalinguistique } \\
(\mathrm{n}=76) \\
12 \% \text { des } \\
\text { rétroactions }\end{array}$ & 0 & $11(14,5 \%)$ & $27(35,5 \%)$ & $\begin{array}{l}50 \% \\
(n=38)\end{array}$ \\
\hline $\begin{array}{ll}\begin{array}{l}\text { Répétition } \\
39)\end{array} \\
6 \% \\
\begin{array}{l} \\
\text { rétroactions }\end{array}\end{array}$ & 0 & $9(23 \%)$ & $15(38 \%)$ & $\begin{array}{l}62 \% \\
(n=24)\end{array}$ \\
\hline $\begin{array}{l}\begin{array}{l}\text { Correction } \\
\text { explicite } \\
37)\end{array} \quad(n= \\
5,8 \% \text { des } \\
\text { rétroactions }\end{array}$ & $11(30 \%)$ & 0 & 0 & $0 \%(n=0)$ \\
\hline 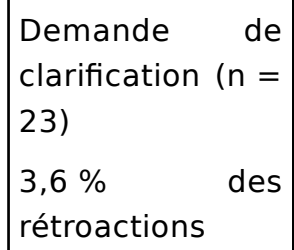 & 0 & $3(13 \%)$ & $5(22 \%)$ & $35 \%(n=8)$ \\
\hline $\begin{array}{l}\text { Totaux : } 635 \\
\text { rétroactions } \\
\text { correctives }\end{array}$ & 70 & 53 & 113 & 166 \\
\hline
\end{tabular}

28 Un regard individuel s'avère nécessaire et montre que le type de rétroaction corrective qui conduit à l'auto et à l'hétéro-réparation est différent selon l'enseignante étudiée. Nathalie utilise le plus fréquemment l'incitation. Si les demandes de clarification et la répétition déclenchent un taux élevé de réparations par les apprenants, leurs occurrences sont faibles ( 2 et 3 occurrences respectivement). Pour Corinne c'est l'incitation qui produit le plus haut degré de réparations chez les apprenants, et si la répétition suit, elle ne fournit ce type de rétroaction que 3 fois. Chez Anne, c'est l'incitation qui conduit au taux plus élevé de réparations générées par les apprenants, suivi par l'indice métalinguistique, qui constitue $18 \%$ de ses rétroactions. Pour Danielle, ce n'est pas l'incitation qui conduit le plus souvent aux réparations générées par les apprenants mais 
l'indice métalinguistique qui représente $15 \%$ de ses rétroactions, suivi par la répétition, qui représente $20 \%$ de ses rétroactions. Finalement, chez Julie, ce sont les répétitions qui induisent le plus de réparations mais seulement 3 occurrences sont trouvées. Ceci est suivi des indices métalinguistiques qui représentent $12,5 \%$ de ses rétroactions.

\section{L'utilisation des prénoms}

En moyenne, les enseignantes observées utilisent entre 46 et 57 fois les prénoms des élèves dans chaque séance observée, à l'exception d'Anne qui a un taux d'utilisation des prénoms significativement plus élevé (tableau 5).

Tableau 5 - L'utilisation des prénoms dans les observations.

\begin{tabular}{|l|l|l|}
\hline Enseignante & Occurrences d'utilisation des prénoms & Nombre de séances observées \\
\hline Nathalie & 243 (moyenne de 49 par séance) & 5 séances \\
\hline Corinne & 275 (moyenne de 46 par séance) & 6 séances \\
\hline Anne & 659 (moyenne de 220 par séance) & 3 séances \\
\hline Danielle & 229 (moyenne de 57 par séance) & 4 séances \\
\hline Julie & 224 (moyenne de 56 par séance) & 4 séances \\
\hline
\end{tabular}

\section{Les occurrences d'encouragement et de dévalorisation des apprenants}

Tableau 6- Les occurrences relevées de valorisation et de dévalorisation des apprenants.

\begin{tabular}{|c|c|c|c|}
\hline Enseignante & $\begin{array}{c}\text { Occurrences } \\
\text { d'encouragement }\end{array}$ & $\begin{array}{c}\text { Occurrences de } \\
\text { dévalorisation }\end{array}$ & $\begin{array}{c}\text { Nombre de séances } \\
\text { observées }\end{array}$ \\
\hline Nathalie & 98 & 9 & 5 séances observées \\
\hline Corinne & 40 & 2 & 6 séances observées \\
\hline Anne & 99 & 2 & 3 séances observées \\
\hline Danielle & 121 & 39 & 4 séances observées \\
\hline Julie & 104 & 21 & 4 séances observées \\
\hline
\end{tabular}

Les valorisations prennent le plus souvent la forme d'énoncés tels que "good" ou "very good" en réponse aux productions des apprenants. Chez Nathalie et Anne, on constate également des moments lors desquels elles encouragent les apprenants à persévérer 
lorsqu'ils rencontrent des difficultés, ou, chez Anne, des moments lors desquels elle les assure que personne ne se moque d'eux.

On constate ici que Danielle et Julie sont celles qui dévalorisent le plus les élèves. La dévalorisation chez Danielle prend le plus souvent la forme d'une sanction, ou bien elle tourne leurs PNCA en dérision.

Ex. Danielle : Des fois je me demande si tu comprends vraiment rien ou si tu écoutes justement strictement rien. On est vraiment pas très sûr des fois.

Ex. A1 : ça veut dire quoi splendid?

Danielle : Splendid?

A2 : Splendide

Danielle : Yes. (ironique) It's really hard

32 Julie se moque également de ce que les élèves ne savent pas et fait des commentaires sur leurs capacités cognitives et compare les élèves entre eux :

Ex. Julie: Quand je pense qu'avant au bac, il y avait des exercices comme ça (rires). C'est fini tout ça.

A1 :Mais pourquoi?

Julie : Ben, parce que vous auriez pas tous la moyenne

Ex. Julie : C'est pas ce que je veux dire que les garçons sont plus forts que les filles hein, mais en l'occurrence dans la classe...

Les deux occurrences repérées chez Anne relèvent de l'étalage de la vie privée d'un apprenant devant la classe et de l'explicitation d'un lien entre le comportement d'un élève et son intelligence.

Ex. Anne (à A1) : Là, tu - tu viens avec moi chez Mme M. Là, ça va pas. On ira voir notre $\mathrm{CPE}$. Tu as été absent depuis deux jours. Ta mère ne vient plus depuis deux semaines. Manifestement, tu es incapable de te débrouiller tout seul. On va t'aider. Tu te tais, tu te calmes, tu te mets au travail !

A1 : Vous voulez même pas m'écouter.

Ex. Anne : Et là, aujourd'hui, tu n'es pas intelligent. Alors, je vais être obligée de sanctionner aujourd'hui.

Chez Nathalie il s'agit des commentaires dévalorisants sur le comportement des élèves et de leur capacité de travail ainsi que l'expression de son énervement lorsqu'ils ne comprennent pas.

Ex. Nathalie : Alors, vos devoirs pour mercredi étant donné que, on n'a pas pu réaliser ce travail à l'oral, vous allez le réaliser à l'écrit et puis, bien sûr ce sera noté étant donné que vous ne travaillez que comme ça.

Ex. A1: Euh, how long ago did you meet?

Nathalie: I euh,so, we met a decade ago. So, ten years ago.

A2 : Ça fait dix ans que vous avez pas vu votre meilleur ami ?

Nathalie : (sur un ton énervé) No. We met. met. Right?

Les deux occurrences de dévalorisation relevées chez Corinne concernent une attitude de dérision à propos des PNCA ou des incompréhensions de la part des apprenants.

Ex. A1 (à un camarade) : Elle vient de te dire quoi ?

Corinne : Qu'est-ce que j'ai dit? Take the diary, ça veut dire quoi?

A2 : Agenda je dirais.

Corinne : Ben oui. C'est pas compliqué, je l'ai répété pendant toute l'année quoi, mais bon. 


\section{Entretiens post-observation et explications a posteriori} taux d'input et le temps de parole accordé aux apprenants. Selon les enseignantes observées qui avait un faible taux d'input (voir tableau 2), les raisons données sont le comportement des élèves (8 occ . Nathalie, Danielle), leur niveau d'anglais jugé insuffisant (7 occ. Nathalie, Corinne, Danielle), le manque de temps pour aller au rythme des apprenants ( 7 occ Nathalie, Corinne) et le sentiment de ne pas savoir faire autrement (2 occ. Corinne, Danielle). Anne et Julie, pour qui les taux d'utilisation de l'anglais sont plus élevés (tableau 2), déclarent que c'est pour favoriser l'acquisition (5 occ.). Anne trouve que la gestion du comportement des élèves est facilitée par l'utilisation de l'anglais (2 occ.). Julie déclare, néanmoins, que si parler l'anglais est son objectif, ce n'est pas toujours possible, pour les points de grammaire par exemple (4 occ.). Corinne ajoute qu'elle parle anglais en cours mais seulement lorsque les apprenants comprennent (1 occ.).

Le faible taux de parole accordé aux apprenants (tableau 3) s'explique par des environnements centrés principalement sur l'enseignant. Une préférence pour un style centré sur l'enseignant s'explique par le sentiment de manquer de temps (11 occ. Nathalie, Corinne, Anne, Danielle, Julie), par l'incapacité perçue des élèves à gérer le travail autonome sur le plan langagier (11 occ. Nathalie, Corinne, Danielle) et / ou sur le plan comportemental (10 occ. Nathalie, Corinne, Julie), ou par le sentiment de ne pas savoir faire autrement (6 occ. Corinne). Il parait pertinent à ce stade de rappeler qu'il s'agit ici de représentations et non de faits.

D'autres explications ont été fournies par mail après que les résultats ont été communiqués aux participantes. Concernant la rétroaction corrective, deux répondantes ont fourni des explications. La raison que Corinne donne pour favoriser la reformulation implicite est le manque de temps ( 2 occ.), pour ne pas entendre un deuxième énoncé " erroné" de la part des apprenants, et parce qu'elle trouve que l'hétéro-correction n'est pas efficace sur le plan acquisitionnel. Anne déclare favoriser la reformulation implicite pour ne pas interrompre la communication (1 occ.).

En ce qui concerne les mesures de valorisation et de dévalorisation des apprenants et l'utilisation de leurs prénoms, 4 des 5 enseignantes ont donné un retour. Nathalie et Anne déclarent utiliser les prénoms des élèves pour que chacun se sente concerné par ce qu'il se passe dans la séance ( 2 occ.). Anne explique également qu'elle estime qu'il est important d'utiliser les prénoms des personnes lorsqu'on s'adresse à elles (1 occ.). Anne se félicite de ne pas se moquer des apprenants et elle déclare s'adresser à ses élèves comme elle voudrait qu'ils s'adressent à elle, c'est-à-dire, "calmement et poliment" (1 occ.). Corinne explique son utilisation plus élevée des prénoms dans certains groupes par l'affinité, par le besoin de connaitre les prénoms dans les grands groupes pour "interroger" les élèves, et par le choix explicite de mettre une barrière dans certains groupes en utilisant moins les prénoms. Elle déclare ne pas se moquer volontairement des apprenants parce qu'elle ne souhaite pas les démotiver. Julie fait remarquer que malgré les difficultés qu'elle a à retenir les prénoms, elle s'intéresse à ses élèves (1 occ.).

Il est à noter qu'une participante (Danielle) n'a plus donné de nouvelles suite à l'entretien post-observatoire. 


\section{Retours réflexifs}

414 des 5 participantes à l'étude ont indiqué que la communication des résultats ainsi qu'une sensibilisation aux théories de l'acquisition et de la psychologie de l'éducation leur ont fait prendre du recul sur leurs pratiques. Nathalie déclare changer la vision qu'elle a des élèves, ce qui était, selon elle, peut-être source de tensions.

Corinne fait état d'une remise en question et d'une modification des comportements concernant ses attentes et sa position comme "transmetteur de savoirs". Elle déclare avoir pris conscience des représentations négatives qu'elle avait de ses élèves et de l'effet Pygmalion produit. Elle déclare également avoir pris conscience du rôle des affects dans l'apprentissage et de la nécessité de s'intéresser aux apprenants et déclare avoir changé sa manière d'interagir avec eux. Néanmoins, elle trouve que cela demande beaucoup d'énergie et elle a l'impression de ne pas avoir assez de temps pour avoir une pédagogie davantage centrée sur les apprenants. Corinne déclare avoir été surprise et vexée par les résultats qui montraient que le français était "très présent" dans les cours observés. Elle déclare que ceci est dû à une impression de manquer de temps mais elle constate qu'il est nécessaire de leur fournir davantage d'input dans la semaine et qu'elle y fait désormais plus attention. Corinne déclare avoir pris peur en prenant conscience du fait qu'elle se moquait des élèves ou était ironique avec eux. Depuis la lecture des résultats portant sur le nombre d'occurrences d'encouragements donnés dans ses groupes, elle déclare faire davantage d'efforts pour encourager les apprenants.

Anne déclare être contente de ne pas pratiquer la correction explicite, car elle s'efforce, dans ses pratiques, d'instaurer l'auto et l'hétéro-correction. Néanmoins, elle trouve qu'il n'y a pas assez de réparations, ce qu'elle pense être dû à sa représentation de ne pas avoir assez de temps, ce qu'elle déclare travailler ( 6 occ.). Quant à son temps de parole, elle déclare vouloir moins intervenir ( 6 occ.). Elle trouve que sa présence au centre de la situation de l'enseignement inhibe l'acquisition.

Julie a indiqué que le regard sur les résultats lui a fait prendre conscience de son style centré sur l'enseignant ( 1 occ.). Elle déclare vouloir essayer de mettre en place d'avantage d'incitation ainsi qu'une approche actionnelle mais qu'elle a "du mal".

\section{Discussion}

Lorsque l'on confronte les observations au cadre théorique, il est possible d'avancer que le temps dévolu à l'anglais est, dans son ensemble, insuffisant pour favoriser l'acquisition langagière (tableau 2). Étant donné les effectifs élevés pour la majorité des groupes observés (tableau 1), la lecture des théories indiquerait que le temps de parole dévolu à chaque apprenant est également insuffisant pour assurer les acquisitions (tableau 3). Ce faible taux de temps de parole disponible aux apprenants s'explique par la nature des activités qui sont, dans l'ensemble centrées sur l'enseignante. Sur l'ensemble des 22 séances, toutes comportaient des activités centrées sur l'enseignante et seulement 7 incluaient des activités centrées sur les apprenants.

Les résultats portant sur les rétroactions correctives montrent que le type de rétroaction utilisé dépend beaucoup de l'enseignante et que la réaction des apprenants aux divers types de rétroaction varie également. Ainsi, un type de rétroaction qui mène à un haut 
degré d'incorporation pour un groupe d'apprenants n'est pas nécessairement le même type de rétroaction qui déclenche un haut degré d'incorporation pour un autre groupe. Ce que cette étude et celle de Lyster \& Ranta (1997) permettent de dégager est que la reformulation implicite est hautement utilisée comme forme de rétroaction et qu'elle ne favorise pas l'incorporation.

En ce qui concerne l'utilisation des prénoms, on constate simplement qu'Anne sort du lot (tableau 5), ce qui, à la lumière des théories présentées (paragraphe 2.2), peut vouloir dire qu'elle valorise hautement les apprenants et peut contribuer à la mise en place d'un environnement propice à l'apprentissage sur le plan affectif.

L'interprétation des occurrences de valorisation et de dévalorisation des apprenants est difficile car la définition des occurrences est subjective, nous n'avons pas accès au ressenti des élèves, et, en ce qui concerne les valorisations, il faudrait un retour des enseignantes pour savoir s'il s'agit d'automatismes ou d'encouragements réfléchis, le premier n'étant pas crédible aux yeux des apprenants. Si les théories ne permettent pas de quantifier un nombre d'occurrences "acceptable" ou "inacceptable", il parait justifié d'avancer que les degrés de dévalorisation trouvés dans les séances de Danielle et de Julie peuvent contribuer à un environnement défavorable à l'acquisition (tableau 6).

Lorsque l'on croise ce qui est observé avec les raisons que donnent les enseignantes pour leurs actions, on constate un effet Pygmalion (Rosenthral \& Jacobsen, 1969; Felouzis, 1997). Les enseignantes qui ont des attentes peu élevées envers les apprenants tendent à ne pas mettre en place des pratiques pédagogiques qui poussent les limites de leurs ZPD (Vygotsky, 1978), réduisant ainsi les chances d'acquisition et donnant finalement raison aux représentations qu'elles se font des capacités des élèves.

Le manque de temps est une raison principale donnée pour ne pas mettre en œuvre des activités qui favorisent l'acquisition. Les participantes indiquent également lors des entretiens le sentiment de ne pas satisfaire aux attentes de l'institution (11 occ.). En effet, il apparait que l'institution exige d'amener les apprenants à un niveau B2 du CECRL sans pouvoir fournir les heures nécessaires pour y parvenir. Par conséquent, les enseignantes ne prennent pas le temps de parler en anglais ni de mettre en place des tâches d'apprentissage qui seraient plus propices à l'acquisition, ni de produire des rétroactions qui permettraient davantage d'incorporation mais préfèrent aller vite pour boucler les programmes. Le résultat est que les apprenants ne sont pas exposés à une quantité d'input suffisante et n'effectuent pas d'activités qui permettraient le traitement en profondeur de cet input ce qui entrave davantage l'acquisition.

51 La gestion du temps se complique davantage lorsqu'elle est croisée avec la gestion du comportement des élèves. La discipline est un élément central dans les discours des enseignantes volontaires observées et parait expliquer en partie leurs choix, notamment l'utilisation du français et la préférence pour un style centré sur l'enseignant. On constate donc l'importance de la prise de recul par rapport aux représentations que l'on construit sur les apprenants que l'on a en face de soi afin de pouvoir mettre en place des pratiques cohérentes avec les théories.

\section{Conclusion}

52 Il semble qu'une analyse des pratiques en lien avec les théories peut participer au développement des retours réflexifs au vu des échanges avec 4 des 5 enseignantes qui ont 
participé à cette étude. Leurs retours conduisent à penser que cette démarche a permis de déstabiliser leurs représentations, ce qui pourra permettre leur évolution. La question se pose alors de réfléchir aux dispositifs qui peuvent être proposés aux enseignants afin de permettre l'évolution des représentations et l'adaptation aux différents contextes d'apprentissage. Une bonne connaissance des théories d'acquisition permettrait à l'enseignant de construire ses cours autrement, en donnant par exemple plus d'occasions aux apprenants de pratiquer, et lui donnerait peut-être plus de souplesse pour s'adapter aux diverses situations qui surgissent sur le terrain et aux conditions de travail réelles. Une bonne connaissance des théories issues de la psychologie de l'éducation pourrait permettre aux enseignants de prendre la mesure de l'impact de leurs attitudes sur l'investissement des apprenants et de prendre du recul par rapport à ce point.

On remarque que c'est le fait que la chercheuse soit allée dans les établissements et ait communiqué les résultats confrontés aux théories qui a déclenché des retours réflexifs sur les pratiques. Il est à noter que sa position n'était ni celle de formatrice ni d'inspectrice mais celle de chercheuse. Ainsi un climat de confiance a pu être instauré entre la chercheuse et des volontaires, a priori prêtes à se remettre en question. Cette modalité d'observation pour amener les enseignants à un retour réflexif est très différente de la formation initiale et des inspections car rien n'est imposé. On permet simplement l'auto-confrontation et la confrontation des pratiques aux théories sans porter de jugement de valeur et sans attitude prescriptive.

Ceci conduit à s'interroger sur le rôle du chercheur en didactique des langues sur le terrain et sur la manière dont il serait envisageable d'articuler davantage la recherche et la pratique, notamment dans l'enseignement secondaire, où les collaborations paraissent rares. Ce travail conduit également à s'interroger sur ce qui peut être mis en place lors de la formation initiale et continue, voire pendant la préparation aux concours pour encourager des retours réflexifs. Cette recherche vient conforter les dispositifs de formation des enseignants qui articulent théories et pratique, dans un va-et-vient constant entre le retour réflexif sur les actions de terrain et la mise en place de nouvelles pratiques en fonction de nouvelles données théoriques.

\section{BIBLIOGRAPHIE}

Bandura, A. (1997). Self-efficacy: The exercise of control. New York: W.H. Freeman.

Bandura, A. (1986). Social foundations of thought and action: A social cognitive theory. Englewood Cliffs, N.J. : Prentice-Hall.

Bardin, L. (2007). L'analyse de contenu. Paris : Presses Universitaires de France.

Brophy, J. E. (1981). "Teachers praise: A functional analysis". Review of Educational. Research, n 51. pp. 5-32.

Burden, P.R. (1995). Classroom management and discipline. New York: Longman.

Chaudron, C. (1988). Second language classrooms. New York: Cambridge University Press. 
Cosereanu-Declerck, E. (2010). Le rôle de la négociation et de la rétroaction corrective dans l'acquisition des langues secondes. La situation des tâches d'interaction en communication synchrone étudiée sur le cas $d u$ français langue étrangère. (Unpublished doctoral dissertation). Université de Technologie de Compiègne.

Demaizière, F., \& Narcy-Combes, J.-P. (2005). "Methodologie de la recherche en didactique : Nativisation, tâches et TIC". Alsic, vol. 8, n 1. pp. 45-64.

De Ribaupierre, A., Ghisletta, P., \& Lecerf, T. (Ed.). (2010). Identité et spécificités de la psychologie différentielle. Rennes: Presses Universitaires de Rennes.

Doughty, C. (1994). "Fine-tuning of feedback by competent speakers to language learners". In Alatis, J. (Ed.). GURT 1993. Washington DC: Georgetown University Press. pp. 96-108.

Doughty, C., \& Williams, J. (Ed.). (1998). Focus on form in classroom second language acquisition. Cambridge : Cambridge University Press.

Dubet, F., \& Martuccelli, D. (1996). A l'école. Sociologie de l'expérience scolaire. Paris : Seuil.

Dubet, F. (2008). Faits d'école. Paris : Editions de l'Ecole des hautes études en sciences sociales.

Duru-Bellat, M. (2006). L'inflation scolaire : les désillusions de la méritocracie. Paris : Editions du Seuil / La république des idées.

Eccles, J.S., Adler, T.F., Futterman, R. Goff, S.B. Kaczala, C.M., Meece, J.L., \& Midgley, C. (1983). "Expectancies, values, and academic behaviors". In Spence J.T. (Ed). Achievement and Achievement motivation. San Fransisco, CA: W.H. Freeman. pp. 75-146.

Elias, N. (1993). Engagement et distanciation. Paris : Fayard.

Ellis, N. C. (2003). "Constructions, chunking, and connectionism: The emergence of second language structure". In Doughty C.J. \& Long M.H. (Eds.). The handbook of second language acquisition . Oxford : Blackwell. pp. 63-103.

Felouzis, G (1997). L'efficacité des enseignants. Paris : Presses Universitaires de France.

Försterling, F., \& Morgenstern, M. (2002). "Accuracy of self-assessment and task performance: Does it pay to know the truth?" Journal of Educational Psychology, $\mathrm{n}^{\circ}$ 94. pp. 76-585.

Goddard, R., Hoy, W. \& Hoy, A. (2004). "Collective efficacy beliefs: Theoretical developments, empirical evidence, and future directions". Educational Researcher, 33(3), pp. 3-13.

Johnstone, K.M., Ashbaugh, H., \& Warfield, T.D. (2002). "Effects of repeated practice and contextual-writing experiences on college students' writing skills". Journal of Educational Psychology, $\mathrm{n}^{\circ}$ 94. pp. 305-315.

Jourdenais, R. (2001). "Cognition, instruction and protocol analysis". In Robinson P. (Ed.). Cognition and Second Language Instruction. Cambride: Cambridge University Press. pp. 354-376.

Lightbown, P., \& Spada, N. (1990). "Focus on form and corrective feedback in communicative language teaching: Effects on second language learning". Second Language Acquisition, $\mathrm{n}^{\circ} 12$. pp. 428-448.

Little, D. (2007). "Language learner autonomy: some fundamental considerations revisited". Innovation in Language Learning and Teaching, vol. 1, n 1. pp. 14-29.

Lyster, R. \& Ranta, L (1997). "Corrective feedback and learner uptake". SSLA, n² 20. pp. 37-66. Mackey, A., Oliver, R., \& Leeman, J. (2003). "Interactional input and the incorporation of feedback: an exploration of NS-NNS adult and child dyads". Language Learning $n^{\circ}$ 53. pp. 35-66. 
Morin, E. (2000). Les sept savoirs nécessaires à l'éducation du futur. Paris : Seuil.

Narcy-Combes, J.-P., \& Narcy-Combes, M.-F. (2007). "La tâche, réponse à des problèmes spécifiques dans le contexte universitaire français". Le Français dans le Monde - Langue et travail pp. 73-86.

Narcy-Combes, J.-P., Narcy-Combes, M.-F. \& Starkey-Perret (2009). "Épanouissement personnel et réussite professionnelle : quelle posture pour l'enseignant ?". LIDIL, nº 4. pp. 139-157.

Norton Pierce, B. (1995). "Social identity, investment, and language learning". TESOL Quarterly, vol. 29, n 1. pp. 9-31.

Pekrun, R. Frenzel, A.C., Goetz, T., \& Perry, R.P. (2007). "The control-value theory of achievement emotions: An integrative approach to emotions in education". In Schutz P.A \& Pekrun R. (Ed.). Emotion in education. Burlington, MA : Elsevier, Academic Press, Educational Psychology Series. pp. 13- 36.

Perry, R.P., Schonwetter, D., Magnusson, J.L., \& Struthers, W. (1994). "Use of explanatory schemas and the quality of college instruction: some evidence for buffer and compensation effects". Research in Higher Education, $\mathrm{n}^{\circ}$ 35. pp. 349-371.

Pica, T. (1994). "Research on negociation: What does it reveal about second-language learning conditions, processes and outcomes". Language Learning, $\mathrm{n}^{\circ}$ 44. pp. 193-527.

Pressley, M., \& McCormick, C. (1995). Cognition, teaching, and assessment. New York: HarperCollins. Raffini, J.P. (1996). 150 ways to increase intrinsic motivation in the classroom. Needham Heights, MA: Allyn and Bacon.

Robinson, P. (Ed). (2002). Individual differences and instructed language learning. Amsterdam: John Benjamins Publishing Company.

Rosenthral, R. \& Jacobsen, L. (1968). "Pygmalion in the Classroom". The Urban Review, vol. 3, nº 1. pp. 16-20.

Schmidt, R. (1990). "The role of consciousness in second language learning". Applied Linguistics, $\mathrm{n}$ - 11. pp. 129-158.

Schmidt, R. \& Frota, S.N. (1986). "Developing basic conversational ability in a second language: A case study of an adult learner of Portuguese". In Day R. (Ed.). Talking to learn: Conversation in second language acquisition. Rowley, MA: New House.

Schunck, D., Pintrich, P. \& Meece, J. (2008). Motivation in education: Theory, research, and applications . Upper Saddle River, NJ: Pearson Education.

Spada, N. \& Fröhlich, M. (1995). COLT: Communicative orientation of language teaching observation scheme: Coding conventions and applications. Sydney, Australia: National Centre for English Language Teaching and Research.

Starkey-Perret, R. (2011). "Posture des enseignants de L2 dans le secondaire : résultats d'une enquête par questionnaire" Les Langues Modernes n 2 / 2011. pp. 75-84.

Sternberg, R.J. \& Williams, W.M. (2010). Educational psychology. Upper Saddle River, NJ: Pearson Education.

Swain, M. (2000). "The output hypothesis and beyond; mediating acquisition through collaborative dialogue". In Lantolf J. (Ed.). Sociocultural Theory and Second Languague Learning. Oxford: Oxford University Press. 
Swain, M. (1995). "Three functions of output in second language learning". In Cook G. \& Seidlhofer B. (Ed.). Principle and practice in applied linguisitcs. Oxford: Oxford University Press. pp. 125-144.

Vygotsky, L.S. (1978). Mind in society: Development of higher psychological processes. Cambridge MA: University of Harvard Press.

Weiner, B. (1992). Human motivation: metaphors, theories and research. Newbury Park, CA: Sage.

Wigfield, A., Eccles, J.S., \& Pintrich, P. (1996). "Development between the ages of eleven and twenty-five". In Berliner D.C. \& Calfee R.C. (Ed.). The handbook of educational psychology. New York : Macmillan. pp. 148-187.

Williams, M., \& Burden, R. (1997). Psychology for Language Teachers. Cambridge : Cambridge University Press.

\section{NOTES}

1. Les prénoms des volontaires ont été changés afin de respecter leur anonymat.

2. Classe d'Accueil

3. Section d'Enseignement Général et Professionnel Adapté

\section{RÉSUMÉS}

Cette contribution propose d'exposer les résultats d'une enquête par observation et par entretien menée auprès de 5 enseignantes d'anglais dans le secondaire. Elle étudie la manière dont les représentations qu'elles construisent sur leur métier influent sur leurs choix pédagogiques et sur leurs attitudes envers les apprenants. Dans un premier temps, les observations sont confrontées aux théories de l'acquisition et de la psychologie de l'éducation. Dans un deuxième temps, on étudiera les explicitations des enseignantes à propos de leur propre action, ce qui permet d'établir quelles représentations ont le plus d'impact sur l'action pédagogique et, par conséquent, sur les potentialités d'acquisition. Finalement, l'étude des échanges avec les participantes, dans l'année qui a suivi l'observation, permet de constater que la confrontation des observations aux théories a favorisé un retour réflexif sur leurs propres pratiques, ce qui dégage des pistes pour la formation des enseignants et pour le rôle des chercheurs en didactique des langues, sur le terrain dans le secondaire.

This article presents results of a qualitative observation and interview study carried out with 5 English teachers in the French secondary school system. The aim of the study is to determine how the teachers' representations of their jobs affect the pedagogical choices they make and the attitudes they have toward learners. First, the classroom observations will be studied through the lens of current theories of acquisition and of educational psychology. Next, the explanations that the teachers themselves give for their classroom actions and behaviors will be studied, thus enabling us to establish which representations have the most impact on pedagogy and, consequently, on acquisition. Finally, it will be shown, through the study of the email exchanges during the year following the classroom observation, how sharing the results of the observation 
analysis along with theories of acquisition and educational psychology destabilized the teachers' representations and led them to reconsider their pedagogy. This opens new doors for teacher training and the role of researchers in the secondary school system.

INDEX

Mots-clés : acquisition, apprenants, attitudes, complexité, Pygmalion, représentations, retours sur investissement, retours réflexifs

Keywords : acquisition, attitudes, complexity, destabilization, learners, Pygmalion, representations, returns on investment

\section{AUTEUR}

REBECCA STARKEY-PERRET

Rebecca Starkey-Perret est docteur en Didactique de l'anglais de l'Université de Nantes où elle enseigne des cours d'anglais de spécialité dans la filière Langues Etrangères Appliquées et intervient en Master Didactique des Langues et des Cultures. Elle est membre du Centre de Recherches sur les Identités Nationales et l'Interculturalité (CRINI). Ses recherches portent sur les représentations des enseignants, sur l'approche par tâches et sur les dispositifs hybrides ainsi que sur le plurilinguisme en milieu institutionnel.

Courriel : Rebecca.Starkey1@univ-nantes.fr

Adresse : Faculté des Langues et des Cultures Etrangères, Chemin la Censive du Tertre - BP 81227, 44312 Nantes Cedex 3 FRANCE 